\title{
Extranodal natural killer/T cell lymphoma with cardiac and abdominopelvic nodular deposits: Unique presentation on 18-fluorodeoxyglucose positron emission tomography computed tomography scan
}

\begin{abstract}
Cardiac lymphoma as secondary extension of non-Hodgkin's lymphoma is described in $30 \%$ of autopsies; however, a few cases are diagnosed in vivo. Primary lymphoma of the heart and pericardium is rare, accounting for $1.3 \%$ of primary cardiac tumors and only $0.5 \%$ of extranodal lymphomas at autopsy. We present a unique case of 68-year-old man diagnosed with extranodal lymphoma with cardiac and abdominopelvic nodular deposits on 18-fluorodeoxyglucose positron emission tomography-computed tomography scan findings.
\end{abstract}

Keywords: 18-fluorodeoxyglucose positron emission tomography-computed tomography scan, abdominopelvic nodular deposit, cardiac lymphoma, natural killer/T cell lymphoma

\section{INTRODUCTION}

Extranodal lymphomas are usually found in the stomach, intestines, nasopharynx, skin, bones, thyroid gland, breasts, lungs, testes, and brain. Primary cardiac lymphoma (PCL) is rare, although reports on the frequency of secondary lymphoma spreading to the heart state a range of $16 \%-28 \%$. ${ }^{11,2]}$ We present a case of $\mathrm{CL}$, in whom the initial diagnosis was probably missed before the patient's pacemaker surgery performed for complete heart block which is one of the clinical presentations of these patients with importance of staging 18-fluorodeoxyglucose positron emission tomography-computed tomography $\left({ }^{18}\right.$ FDG PET-CT) scan.

\section{CASE REPORT}

A 68-year-old man with a history of idiopathic thrombocytopenic purpura (postsplenectomy and on treatment) was treated with antibiotics and antipyretics for pyrexia of unknown origin (PUO). He further underwent pacemaker placement for complete heart block. After an interval of $1 \frac{1}{2}$ months, he presented with fever with chills

\begin{tabular}{|l|c|}
\hline \multicolumn{2}{|c|}{ Access this article online } \\
\hline \multirow{2}{*}{$\begin{array}{l}\text { Website: } \\
\text { www.asjo.in }\end{array}$} & Quick Response Code \\
\hline DOI: & \\
10.4103/ASJO.ASJO_89_16 & \\
\hline
\end{tabular}

and two-dimensional (2D) echo color Doppler revealed large echo dense mass in AV groove measuring $22.6 \mathrm{~cm}^{2}$ and a left atrial mass possibly myxoma/sarcoma with mild to moderate pleural effusion for which he was referred for a whole body ${ }^{18} \mathrm{FDG}$ PET-CT scan. The scan demonstrated a large area of irregular uptake involving soft tissue nodular masses in interatrial septum and left atrium extending into mitral valvular region, and possibly tricuspid valvular region, extending in interventricular septum and further into aortopulmonary recess, also extending along posterior wall of right atrium superiorly between region of pulmonary

\section{Shimpi Madhuri Mahajan, Sudeshna MaItra, Natasha Singh, Melvika Pereira}

Department of Nuclear Medicine and PET-CT, P.D. Hinduja National Hospital and MRC, Mumbai, Maharashtra, India

Address for correspondence: Dr. Shimpi Madhuri Mahajan, Department of Nuclear Medicine and PET-CT, P.D. Hinduja National Hospital and MRC, V S Marg, Mahim (W), Mumbai - 400 016, Maharashtra, India.

E-mail: docmadhurim@gmail.com

This is an open access article distributed under the terms of the Creative Commons Attribution-NonCommercial-ShareAlike 3.0 License, which allows others to remix, tweak, and build upon the work non-commercially, as long as the author is credited and the new creations are licensed under the identical terms.

For reprints contact: reprints@medknow.com

How to cite this article: Mahajan SM, Maitra S, Singh N, Pereira M. Extranodal natural killer/T cell lymphoma with cardiac and abdominopelvic nodular deposits: Unique presentation on 18-fluorodeoxyglucose positron emission tomography computed tomography scan. Asian J Oncol 2017;3:74-7. 
trunk and superior vena cava (maximum standard uptake value [SUVmax] 12.2) with irregular linear uptake along region of aortic arch [Figures 1, 2a and b]. There was mild pericardial effusion. Multiple nodular soft tissue deposits [Figure 3a and b] were seen in the abdominopelvic region which were predominantly at the end of the vessels. Nodular lesion seen measuring approximately $1.8 \mathrm{~cm} \times 1.5 \mathrm{~cm}$ medial to left adrenal gland abutting left renal vessels (SUVmax 8.9), lesion abutting left renal pelvis (SUVmax 8.6), near left posterior upper abdomen wall (SUVmax 8.3) and bilateral iliac fossa (SUVmax 5.4). There was a focal uptake seen in thickening involving abdominal aorta at the level of renal

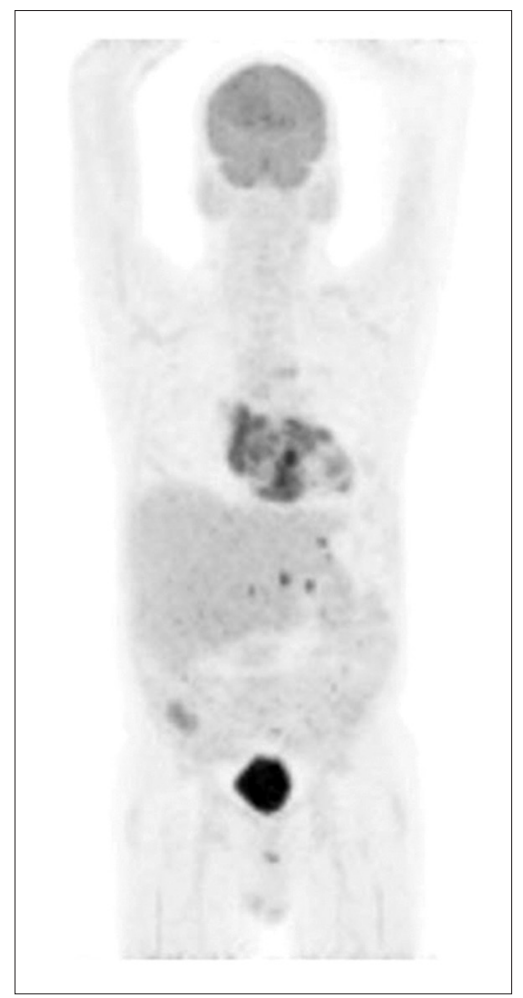

Figure 1: Maximum intensity projection images showing abnormal 18-fluorodeoxyglucose uptake in cardiac and abdominopelvic region vessels (SUVmax 7.4) and also a curvilinear focal uptake in sacral neural foramina on the left side (SUVmax 5.6).

CT-guided biopsy from a deposit near the left posterior upper abdominal wall was suggestive of non-Hodgkin's lymphoma (NHL; peripheral natural killer/T-cell lymphoma). The cells showed strong cytoplasmic positivity for CD3, CD2, CD56, T1a, and negative for CD5, CD7, CD4, CD8, CD30, NK1. Epithelial membrane antigen was negative, and Epstein-Barr virus RNA was equivocal. The final diagnosis was NHL stage IV (heart and abdomen). He received 1 cycle of cyclophosphamide, doxorubicin, vincristine, and prednisone chemotherapy, after which he succumbed to death due to complication secondary to low platelet counts.

\section{DISCUSSION}

Cardiac tumors are classified as primary benign or malignant tumors that arise from the heart, or as secondary metastatic tumors that invade the heart. Primary cardiac tumors occur with a low incidence. It is estimated that secondary metastatic tumors are a hundred times more common than primary cardiac lesions.

$\mathrm{CL}$ as a secondary extension of NHL is described in $30 \%$ of autopsies; ${ }^{[3]}$ however, few cases are diagnosed in vivo. Of all primary cardiac tumors, lymphomas are rare, especially among immunocompetent individuals (1.3\%) and only $0.5 \%$ of extranodal lymphomas at autopsy ${ }^{[4,5]} \mathrm{PCL}$ is a rare clinical entity, The frequency of secondary lymphoma spreading to the heart state a range $16 \%-28 \% .{ }^{[1]}$ Curtsinger et al. ${ }^{[6]}$ reported 15 cases in 1989, and Nakchbandi reviewed 29 more cases in 1995. In the latter report, there were 6 cases related to AIDS. In the report of 15 cases by Curtsinger et al., only 2 cases were diagnosed with PCL before death. It was indicated that most PCL cases were diagnosed at autopsy. However, because of advances in diagnostic methods, such cases are now mostly diagnosed
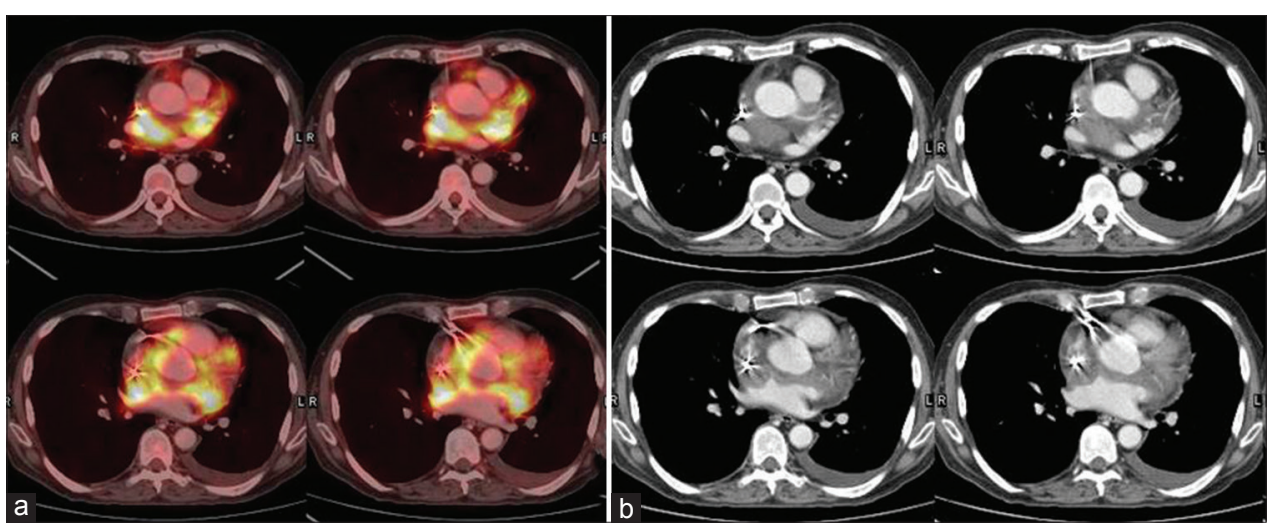

Figure 2: ( $\mathrm{a}$ and b) Fused positron emission tomography-computed tomography and computed tomography images: Large area of irregular uptake involving soft tissue nodular masses in interatrial septum and left atrium extending into mitral valvular region, and possibly tricuspid valvular region 


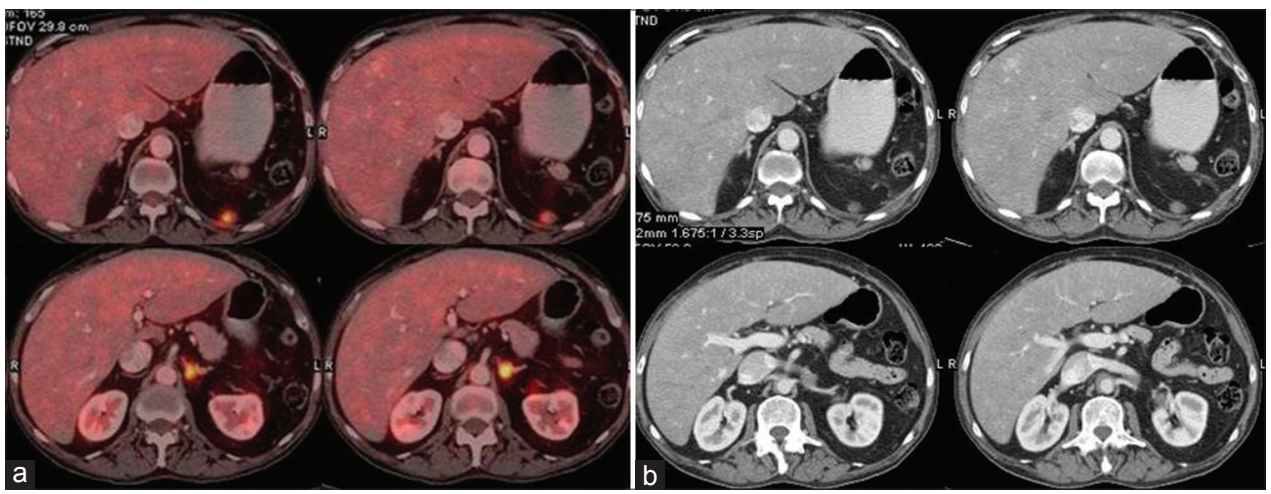

Figure 3: (a and b) uptake in multiple nodular soft tissue deposits in abdomen pelvis

before death. ${ }^{[7]}$ More than $80 \%$ of PCLs are noted as diffuse B-cell lymphomas, mostly with a large cell type. However, the incidence of NHL natural killer/T-cell type is scarce which was the histology of our case with prodigious abdominopelvic soft tissue deposits. Patients with PCL range in age from 18 to 77 years with equal sex distribution. The more common symptoms of cardiac involvement include dyspnea, congestive heart failure, pericardial effusion, and hemopericardium. Cardiac arrhythmias and nonspecific electrocardiographic abnormalities are also common, notably atrioventricular blocks ranging from first degree to third degree. They commonly manifest as an ill-defined, infiltrative mass in the myocardium, often with an associated pericardial effusion.

They can result from the retrograde lymphatic spread, hematogenous spread, or direct extension from adjacent masses. ${ }^{|8|}$ The most common appearance of $\mathrm{CL}$ is hematogenous spreading into the interatrial septum followed by infiltration of cardiac structures by contiguous lymphoma of the mediastinum. These tumor usually extend along the epicardial surfaces of the heart, primarily encasing adjacent structures including coronary arteries and the aortic root. When infiltration beyond the myocardium occurs, the right atrium is most commonly involved. It is an oncologic emergency due to its rapid evolution and advanced stage of organ infiltration. Treatment most commonly includes anthracycline-based chemotherapy and anti-CD20 treatment. Chemotherapy has been used alone or in combination with radiation therapy. Palliative surgery has been performed, mainly for tumor debulking. The prognosis for patients with either primary or secondary lymphomatous heart involvement is usually poor; late diagnosis is one of the major factors affecting the outcome.

The diagnostic modalities such as echocardiography and CT scans are less useful in the early diagnosis of cardiac lesions. ${ }^{\mid 9]}$ In CT, the attenuation of $\mathrm{CL}$ may be similar to or lower than that of normal myocardium. At magnetic resonance imaging (MRI), it has the variable signal intensity and contrast enhancement. ${ }^{[10]}$ PET-CT has gained favor as an imaging modality to assess tumor extent and response to chemotherapy and to predict the risk of disease relapse in patients with lymphoma. However, it is important to understand the normal patterns of cardiac FDG activity that can be seen in oncologic ${ }^{18}$ FDG PET-CT studies. ${ }^{[11]}$ These include focal and regional patterns of increased FDG myocardial activity. Focal activity can be observed in papillary muscles, the atria, the base, and the distal anteroapical region of the left ventricle. Regional increased cardiac FDG activity may be diffuse or localized in the posterolateral wall or the base of the left ventricle. Abnormal patterns of cardiac FDG activity not related to malignancy include those associated with lipomatous hypertrophy of the interatrial septum, epicardial and pericardial fat, increased atrial activity associated with atrial fibrillation or a prominent crista terminalis, cardiac sarcoidosis, endocarditis, myocarditis, and pericarditis. Knowledge of these patterns of cardiac FDG activity is important to be able to recognize malignant disease involving the paracardiac spaces, myocardium, and pericardium.

${ }^{18}$ FDG PET-CT may have the advantage of detecting the tumor or metastases at an early stage, and in cases such as the one described above, it may help in the differentiation between benign and malignant lesions of the heart, which is difficult with other imaging modalities. The unparalleled feature of ${ }^{18}$ FDG PET-CT in detecting malignant lesions due to its high hypermetabolism, helps even the smaller lesions in addition to staging extranodal lymphoma and also modify the disease management. This has high value when lesions are located in the ventricular myocardium with large intracavitary masses because it enables to differentiate neoplastic masses from thrombi. We also emphasize the fact that Integration of ${ }^{18} \mathrm{FDG}$ PET-CT into lymphoma staging and monitoring facilitates the detection of cardiac involvement and the assessment of response to therapy. Serial PET has been suggested to be more accurate than MRI and echocardiography for assessing CL regression. ${ }^{[12]}$ 


\section{Financial support and sponsorship}

Nil.

\section{Conflicts of interest}

There are no conflicts of interest.

\section{REFERENCES}

1. Sarjeant JM, Butany J, Cusimano RJ. Cancer of the heart: Epidemiology and management of primary tumors and metastases. Am J Cardiovasc Drugs 2003;3:407-21.

2. Lepeak LM, Yang DT, Chang JE. Extranodal NK/T-cell lymphoma presenting with primary cardiac involvement. Hematol Rep 2011;3:e9.

3. Gowda RM, Khan IA. Clinical perspectives of primary cardiac lymphoma. Angiology 2003;54:599-604.

4. Burke A, Virmani R. Tumors of the heart and great vessels. In: Atlas of Tumor Pathology. $3^{\text {rd }}$ Series, Fascicle 16. Washington, DC: Armed Forces Institute of Pathology; 1996.

5. Burke A, Jeudy J Jr., Virmani R. Cardiac tumours: An update: Cardiac tumours. Heart 2008;94:117-23.

6. Curtsinger CR, Wilson MJ, Yoneda K. Primary cardiac lymphoma. Cancer 1989;64:521-5.

7. Bishop WT, Chan NH, MacDonald IL, Tutassaura H. Malignant primary cardiac tumour presenting as superior vena cava obstruction syndrome. Can J Cardiol 1990;6:259-61.

8. Guermazi A, Brice P, de Kerviler EE, Fermé C, Hennequin C, Meignin V, et al. Extranodal Hodgkin disease: Spectrum of disease. Radiographics 2001;21:161-79.

9. Kanesvaran R, Tao M, Huat IT, Weng DT, Eng DN, Thye LS. Malignant arrhythmia: A case report of nasal NK/T-cell lymphoma with cardiac involvement. Acta Oncol 2009;48:637-9.

10. Mallia A, Travaini L, Trifiro G, Paganelli G. Detection of a cardiac mass by [18F] FDG-PET/CT: A rare case. Ecancermedicalscience 2009;3:152.

11. Maurer AH, Burshteyn M, Adler LP, Steiner RM. How to differentiate benign versus malignant cardiac and paracardiac 18F FDG uptake at oncologic PET/CT1. Radiographics 2011;31:1287-305. Doi: 10.1148/ rg.315115003.

12. Mato AR, Morgans AK, Roullet MR, Bagg A, Glatstein E, Litt HI, et al. Primary cardiac lymphoma: Utility of multimodality imaging in diagnosis and management. Cancer Biol Ther 2007;6:1867-70. 